\title{
Multidisciplinary Optimization for LHD Working Device Based on Isight
}

\author{
Zhao Lei", Ma Fei, Gu Qing and Shen Yanhua
}

University of Science and Technology Beijing, Beijing, 100083, P.R. China

\begin{abstract}
The design of the LHD working device is a complex problem, often related to multi-variables, multi-objective, nonlinear constraint. In this paper, MDO theory is proposed with $14 \mathrm{t}$ wheel loader as research target and Isight is as the multidisciplinary optimization platform combined Matlab, Solid work sand Simulation. The mathematical model of working device is established based on Matlab, and single disciplinary optimization of kinematic is executed with the application of genetic algorithms. The study includes: setting up the finite element model under an Ansys Workbench to execute a single disciplinary multiple goal driven optimization on stiffness and mass target; comparison of the results of multidisciplinary and single disciplinary optimization. The analysis result indicates that this platform enables the combination of experimental design and multi-objective optimization to automatically calculate, build modeling and simulate. The obtained Pareto graph and Pareto front graph could provide data support for designer's appropriate optimal selection. Compared with the single disciplinary optimization, the bucket tilt force multiplication coefficient is increased by $1.5 \%$ and boomer mass decreased, by $18 \%$.
\end{abstract}

Keywords: Integration based on Isight, LHD, Multidisciplinary Optimization, Working Device.

\section{INTRODUCTION}

In the field of mining machinery, objects design are usually very complicated, typically involving multiple disciplines, such as structural strength, mass, kinematics, vibration, and performance. There are respective unique local variables among various sub-disciplines. The coupling between these sub-disciplines is realized through sharing global variables. In this case, the mathematical description of the design object is a very enormous nonlinear optimization problem. The traditional design approach generally individually optimizes each subject and then combines the optimal portions to get the final optimization result. Reference [1] optimized the loader working device from the translation and force transferring ratio. Reference [2] optimized bucket trajectory, boom dip angle and digging force of loader working device. Reference [3] build three-dimensional model bommer and cylinder by Pro/E and made static strength calculation by Ansys.

Currently, the single disciplinary optimization of each sub-discipline is relatively mature, but these methods do not consider the coupling between sub-disciplines. Therefore, optimization results are not reasonable. In view of this, this article introduces multidisciplinary optimization (MDO) to solve such problems. Multidisciplinary design optimization is a theory to design complex product and its subsystems by exploring and utilizing its cooperation mechanism. J. Soboeszczanski-Soboeski first proposed MDO theory about solving large-scale structural optimization problems [4], then MDO theory was further elaborated in his series of literature $[5,6]$. Boeing Company described the MDO optimized

*Address correspondence to this author at the University of Science and Technology Beijing, Beijing, China; E-mail: zl7970@126.com method between behavior, performance, ergonomics and reliability, studied approximate models. Reference [7] proposed the application method of MDO in the optimization of jet plane wing shape.

Meanwhile, in order to provide visibility model support, here the author utilize Isight, the software of multidisciplinary optimization.

Multidisciplinary optimization software Isight packages with a variety of optimization algorithms to integrate multiple software modules. It provides a visualization platform with automatic modeling, automatic analysis and automatic calculation, which facilitate real-time monitoring of the entire process by engineers [8].

\section{KINEMATICS OPTIMIZATION MODEL}

\subsection{Model Analysis}

Underground LHD is designed primarily considering five kinds of conditions: insert condition, shoveling condition, lifting condition, high unloading condition and low unloading condition [9]. The front frame is selected here as the reference coordinates system, and in shoveling conditions, position coordinates of working device's each hinge point is selected as optimized design variables, so as to establish the work equipment mechanism diagram as shown in Fig. (1). During the optimization, the main consideration is tilt force multiplication coefficient, without considering the impact generated from hinge point coordinates of lifting mechanism composed of boomer and lift cylinder on optimization goals [10]. 


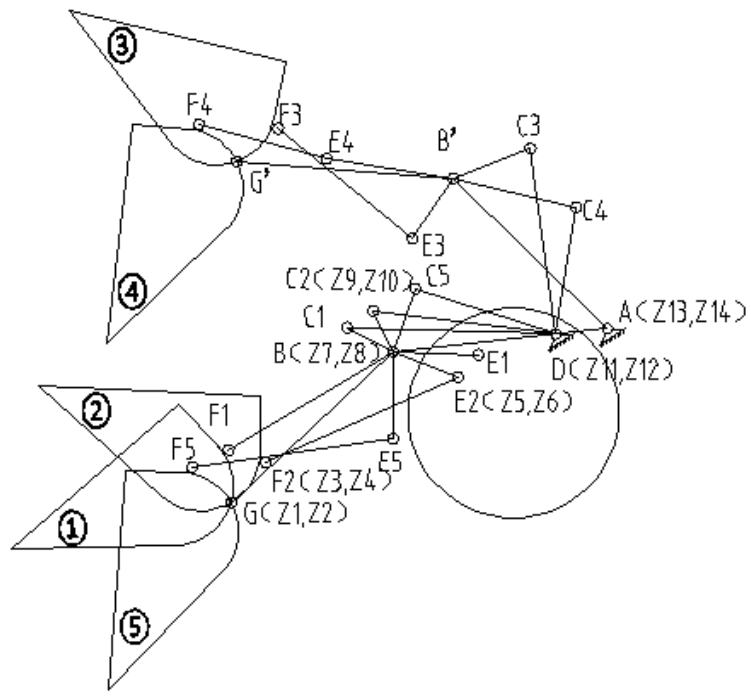

1-insert condition, 2-shoveling condition, 3-lifting condition, 4-high unloading condition, 5-low unloading condition

Fig. (1). Schematic diagram of 5 working cases in device loading process.

Table 1. Initial design requirements of wheel loader.

\begin{tabular}{|c|c|c|}
\hline Parameter & Parameter Value & Measure \\
\hline \hline Bucket capacity & 5.4 & $\mathrm{~m}$ \\
\hline Bucket dead weight & 14 & $\mathrm{t}$ \\
\hline lifting force & 275 & $\mathrm{kN}$ \\
\hline Tilt shovel power & 230 & $\mathrm{~km}$ \\
\hline Discharge distance & 2137 & $\mathrm{~mm}$ \\
\hline Discharge height & 2400 & $\mathrm{~m}$ \\
\hline
\end{tabular}

\subsection{Initial Model of Working Device}

According to the parameters of $14 \mathrm{t}$ LHD on the market, related dimensions are redesigned. Bucket capacity is $5.4 \mathrm{~m}^{3}$, bucket loading capacity is $14 \mathrm{t}$. Specific initial size is shown in Table 1.

\subsection{Design Variables}

During shoveling condition, each hinge point's position coordinates of the link mechanism is set as optimized design variables, shown with vector representation $\vec{X}$ as follows:

$$
\begin{aligned}
& \vec{X}=\left[z_{1}, z_{2}, z_{3}, \ldots, z_{14}\right]^{T} \quad \mathrm{~g}_{1}(\overrightarrow{\mathrm{X}})=\left|\alpha_{\mathrm{i}}-\alpha_{2}\right|-10^{\circ} \leq 0 \\
& \overrightarrow{\mathrm{X}}=\left[\mathrm{z}_{1}, \mathrm{z}_{2}, \mathrm{z}_{3}, \ldots, \mathrm{z}_{14}\right]^{\mathrm{T}}
\end{aligned}
$$

\subsection{Objective Function}

When tilt equipment has a large force transmission, working device's dynamic performance is relatively better. Therefore, the objective function improves tilt force multi- plication coefficient on the basis of meeting the LHD working performance, as shown in Fig. (2).

$$
\min F(\vec{X})=K_{Z B}=\frac{l_{2} \times l_{4}}{l_{1} \times l_{3}}
$$

Wherein: $l_{1}=2393 \mathrm{~mm}$ is the length of bucket bottom, $\mathrm{l}_{2}$ is the vertical distance from $\mathrm{G}$ to $\mathrm{EF}, \mathrm{l}_{3}$ is the vertical distance from $\mathrm{B}$ to $\mathrm{EF}, \mathrm{l}_{4}$ and is the vertical distance from $\mathrm{B}$ to $\mathrm{DC}$ action line.

\subsection{Constraint Equation}

\subsubsection{Parallel Motion Requirements of Bucket Lifting Process}

$$
g_{1}(\vec{X})=\left|\alpha_{i}-\alpha_{2}\right|-10^{\circ} \leq 0
$$

Wherein : $\alpha_{\mathrm{i}}$ is the angle between bucket and ground in lifting process, $\alpha_{2}$ is the angle between bucket and ground in 


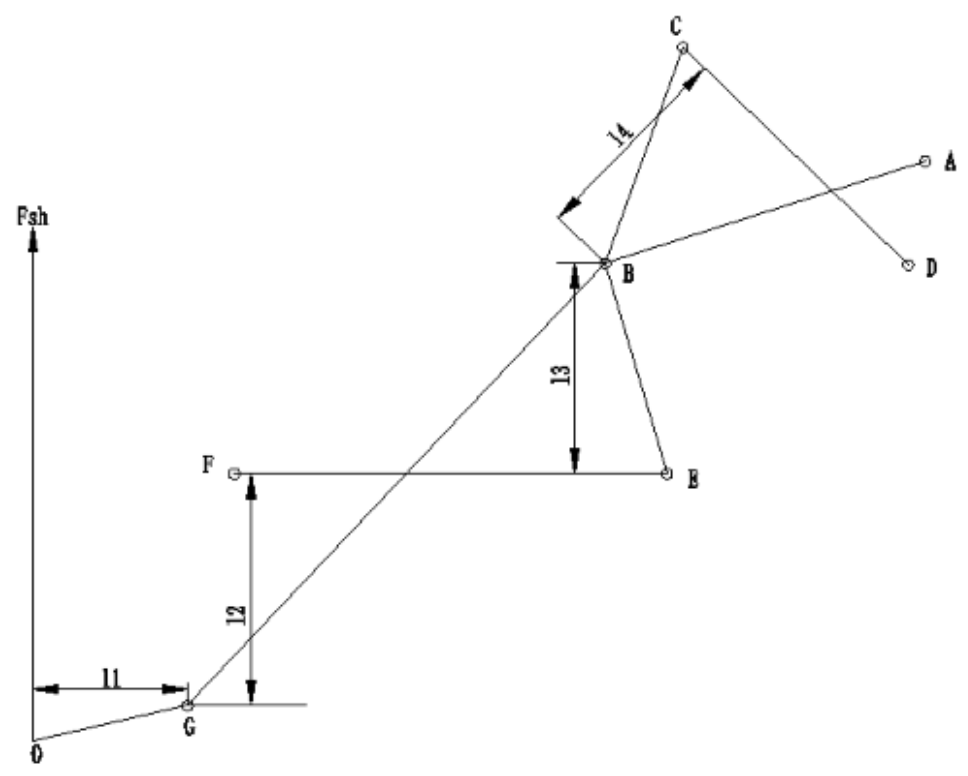

Fig. (2). Schematic diagram of the bucket tilt force.

shoveling condition. $\alpha_{i}=f\left(\vec{X}, \gamma_{i}\right), \quad \gamma_{i}$ is the instantaneous

angle of boom movement. From shoveling condition to lifting condition (as shown in Fig. 2), boom angle changes from $\gamma_{2}$ to $\gamma_{3}$, setting $\gamma_{2}=0, \gamma_{3}=85^{\circ}[11]$.

\subsubsection{Unloading Constraint}

From shoveling condition to lifting condition (as shown in Fig. 2), there is no change in tilt cylinder length, but change in $\alpha_{i}$ caused by boom angle [12]. From lifting condition to high unloading condition (shown as Fig. 2), there is no change in boom angle, but change in $\alpha_{\mathrm{i}}$ caused by tilt cylinder length change. There should be :

$g_{2}(\vec{X})=\alpha_{4}-\alpha_{1}-\varphi_{4}-\alpha_{0}$

Wherein $\varphi_{4}$ is bucket high unloading angle. Set $\varphi_{4} \geq 45^{\circ}[13] ; \alpha_{0}$ is the angle between bucket and ground in shoveling condition. $\alpha_{0}=9^{\circ}$.

\subsubsection{Maximum Unloading Height and Minimum Unload- ing Distance}

Unloading Height:

$g_{3}(\vec{X})=-h_{X}=h_{\max }-y_{1}^{\prime}+O G \sin \varphi_{O_{4} G} \leq 0$

Unloading Distance:

$g_{4}(\vec{X})=l_{\min }-x_{q}+R-x_{1}^{\prime}-O G \cos \varphi_{O_{4} G} \leq 0$

Wherein: $\mathrm{OG}$ is the distance from $\mathrm{O}$ to $\mathrm{G}, \mathrm{x}_{\mathrm{q}}$ is the center coordinates of front wheel, $\mathrm{R}$ is tire static radius, $\varphi_{\mathrm{O}_{4} \mathrm{G}^{\prime}}$ angle between $\mathrm{OG}$ and ground in high unloading condition, $\varphi_{\mathrm{O}_{4} \hat{\mathrm{G}}}=\varphi_{4}+\arctan \frac{\mathrm{y}_{1}}{\mathrm{x}_{1}}-8^{\circ}$.

\subsubsection{Unloading Angle}

If unloading angles of the working device in both high and low unloading conditions are greater than $45^{\circ}$, discharge at each location could be successful. From (Fig. 2) we can see if it satisfies automatic leveling conditions, it must meet unloading angle requirements in high unloading conditions. Therefore, it only needs to consider low unloading condition.

Unloading angle of low unloading condition [14]:

$g_{5}(\vec{X})=\varphi_{5}=45^{\circ}-\alpha_{5}+\alpha_{1}+\alpha_{0} \leq 0$

Meanwhile, considering cylinder's stability requirements, there is

$g_{6}(\vec{X})=\frac{C D}{C D_{5}}-1.6 \leq 0$

\subsubsection{Transmission Angle Constraint}

During LHD operation process, in order to avoid the occurrence of working device's "self-locking" and enhance transmission efficiency [15], it requires the angle between bucket cylinder and rocker arm, and the angle between connecting rod and bucket changing within $10^{\circ} \sim 170^{\circ}[16]$.

$g_{7}(\vec{X})=\angle E F G-170^{\circ} \leq 0$

$g_{8}(\vec{X})=\angle D C B-10^{\circ} \leq 0$

$g_{9}(\vec{X})=\angle D C_{4} B-170^{\circ} \leq 0$

$g_{10}(\vec{X})=\angle E_{4} F_{4} G-10^{\circ} \leq 0$ 


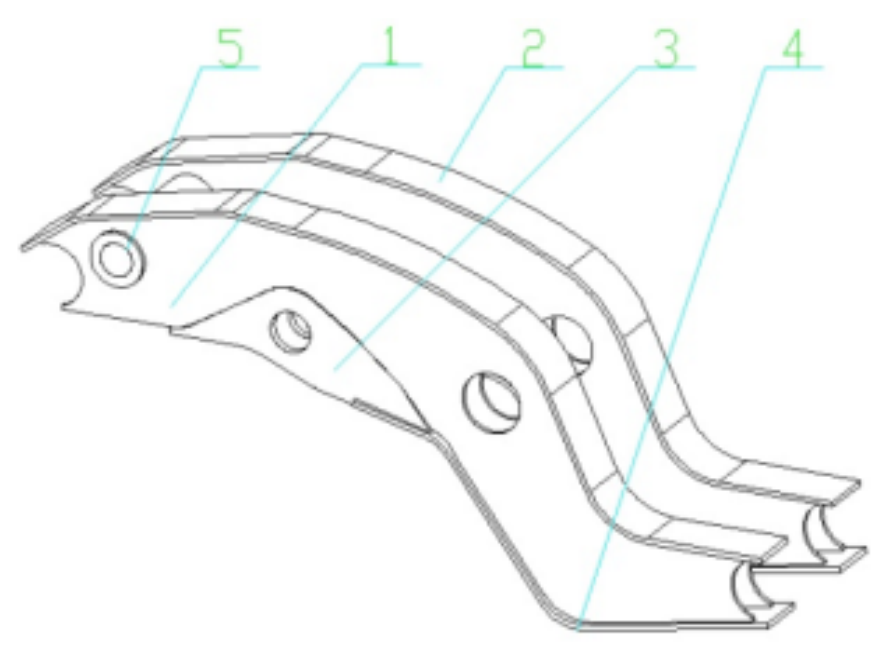

1-Side plate, 2-Roof, 3-lifting oil cylinder hinged ear plate, 4-Floor, 5-upper beam

Fig. (3). Boomer structure diagram.

\subsubsection{Coordination Constraints}

$g_{11}(\vec{X})=L_{G F}+L_{G B}-L_{B E}-L_{E F} \leq 0$

$g_{12}(\vec{X})=L_{B E}+L_{G B}-L_{E F}-L_{G F} \leq 0$

$g_{13}(\vec{X})=L_{G F}+L_{E F}-L_{G E} \leq 0$

$g_{14}(\vec{X})=L_{B E}+L_{E F}-L_{F B} \leq 0$

$g_{15}(\vec{X})=L_{B C}+L_{B A}-L_{C_{1} D}-L_{A D} \leq 0$

$g_{16}(\vec{X})=L_{A D}+L_{B A}-L_{C_{1} D}-L_{B C} \leq 0$

$g_{17}(\vec{X})=L_{B C}+L_{C_{1} D}-L_{B D} \leq 0$

\subsubsection{Boundary Constraints}

$g_{u}(\vec{X})=z_{v}-A_{v} \leq 0 \quad(v=1,2,3, \ldots, 14 ; u=18,19, \ldots, 31)$

$g_{u}(\vec{X})=B_{v}-z_{v} \leq 0 \quad(v=1,2,3, \ldots, 14 ; u=32,33, \ldots, 45)$

\subsection{Optimized Mathematical Model Based on the Sensi- tivity Method}

Link mechanism optimization model:

$$
\begin{aligned}
& \operatorname{Min} F(\vec{X})=-K_{Z B} \\
& \text { s.t } g_{u}(\vec{X}) \leq 0 \quad(u=1,2,3, \ldots, 45)
\end{aligned}
$$

\section{OPTIMIZATION MODEL OF STIFFNESS}

The boomer adopts box-type structure which has better stiffness than the traditional board structure, as shown in Fig. (3).

By using response surface methodology, the thickness dimension of the boomer is optimized by the Goal Driven Optimization module of Ansys Workbench [17].

\subsection{Determination of Working Load}

The external load of bucket is reduced to horizontal load and vertical load.

Horizontal load:

$R_{x}=F_{K}-F_{f}=\frac{M_{q}}{r_{d}}-G_{s} f$

The vertical load

$R_{y}=\frac{L_{1} G_{s}}{L_{2}}$

Wherein: $F_{K}$ is traction of wheel loader. $F_{f}$ is friction of the driven wheel. $M_{q}$ is torque of the driven wheel. $r_{d}$ is radius of the tire.

Unbalanced loading working condition is the studying condition. Under the unbalanced loading working condition, eccentric load suffered by the bucket is equivalent to the point force and additional couple in the bucket center [18]. The analysis of point force under symmetric load condition is a method that various components of work equipment are split into separated bodies, such bodies as the bucket, rod, rocker arm and boom, are successively taken to solve out force, as shown in Fig. (4).

To bucket: 


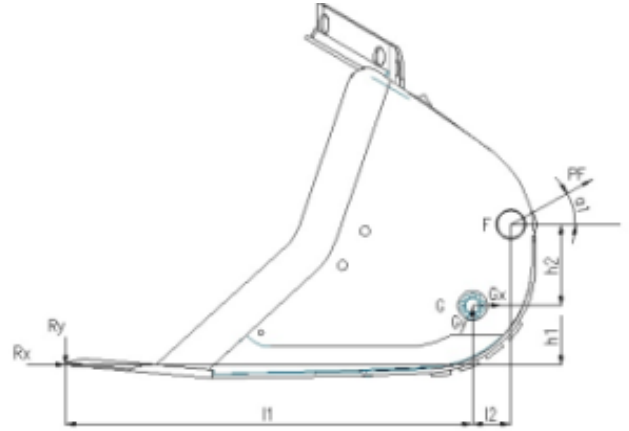

(a) Bucket

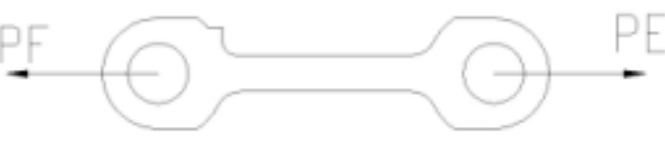

(b) Dog bone

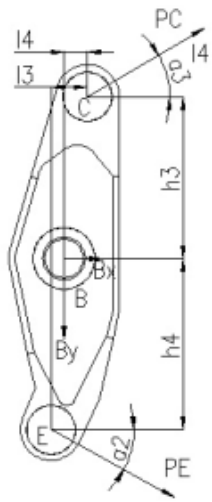

(c) rocker arm

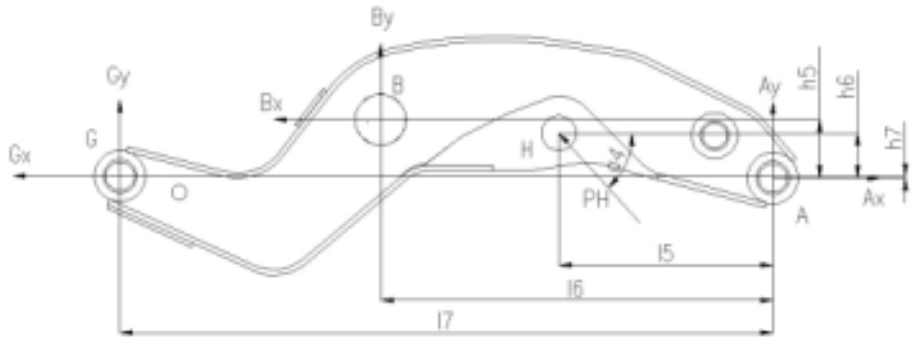

(d) Boomer

Fig. (4). Stress diagram of working device.

$\sum M_{B}=0, P_{c}=\frac{R_{X}^{a} h_{1}+R_{Y}^{a} l_{1}}{h_{2} \cos \alpha_{1}+l_{2} \sin \alpha_{1}}$

$\sum F_{X}=0, X_{B}=-\left(R_{X}^{a}+P_{c} \cos \alpha_{1}\right)$

$\sum F_{Y}=0, Y_{B}=-\left(R_{Y}^{a}+P_{c} \sin \alpha_{1}\right)$

To dog bone: $P_{F}=P_{E}$

To rocker arm:

$\sum X=0, X_{G}=P_{F} \cos \alpha_{2}+P_{E} \cos \alpha_{3}$

$\sum Y=0, Y_{G}=P_{E} \sin \alpha_{3}-P_{F} \sin \alpha_{2}$

$\sum M_{G}=0, P_{E}=P_{F} \frac{h_{3} \cos \alpha_{2}-l_{3} \sin \alpha_{2}}{h_{4} \cos \alpha_{3}-l_{3} \sin \alpha_{3}}$

To boomer:

$\sum M_{A}=0, P_{H}=\frac{X_{G} h_{5}+Y_{G} l_{6}+X_{B} h_{7}+Y_{B} l_{7}}{h_{6} \cos \alpha_{4}+l_{5} \sin \alpha_{4}}$

$\sum X=0, X_{A}=X_{G}+X_{B}+P_{H} \cos \alpha_{4}$ $\sum Y=0, Y_{A}=-\left(Y_{G}+Y_{B}+P_{H} \sin \alpha_{4}\right)$

Set the load capacity as 14 ton. According to static equivalence principle, the force is distributed in bucket with $1000 \mathrm{~mm}$ from the middle.

\subsection{Establishing Mathematical Model}

Set the thickness of side plate as $t_{1}$, of roof $t_{2}$, of the lower plate $t_{3}$, and of the lift cylinder hinge plate $t_{4}$. Set the thickness of upper beam plate as design variable $t_{5}$ (as shown in Fig. 3). $\vec{T}=\left[t_{1}, t_{2}, t_{3}, t_{4}, t_{5}\right]^{T}$.

The objective function is the mass of whole boomer:

$W(\vec{T})=\sum_{j=1}^{n} m_{j}$

In the formula: ' $\mathrm{n}$ ' is cell number, $m_{j}$ is mass of the $\mathrm{j}$-th cell.

\subsubsection{Constraints}

The maximum equivalent stress requiring the allowable stress is less than Q345: 


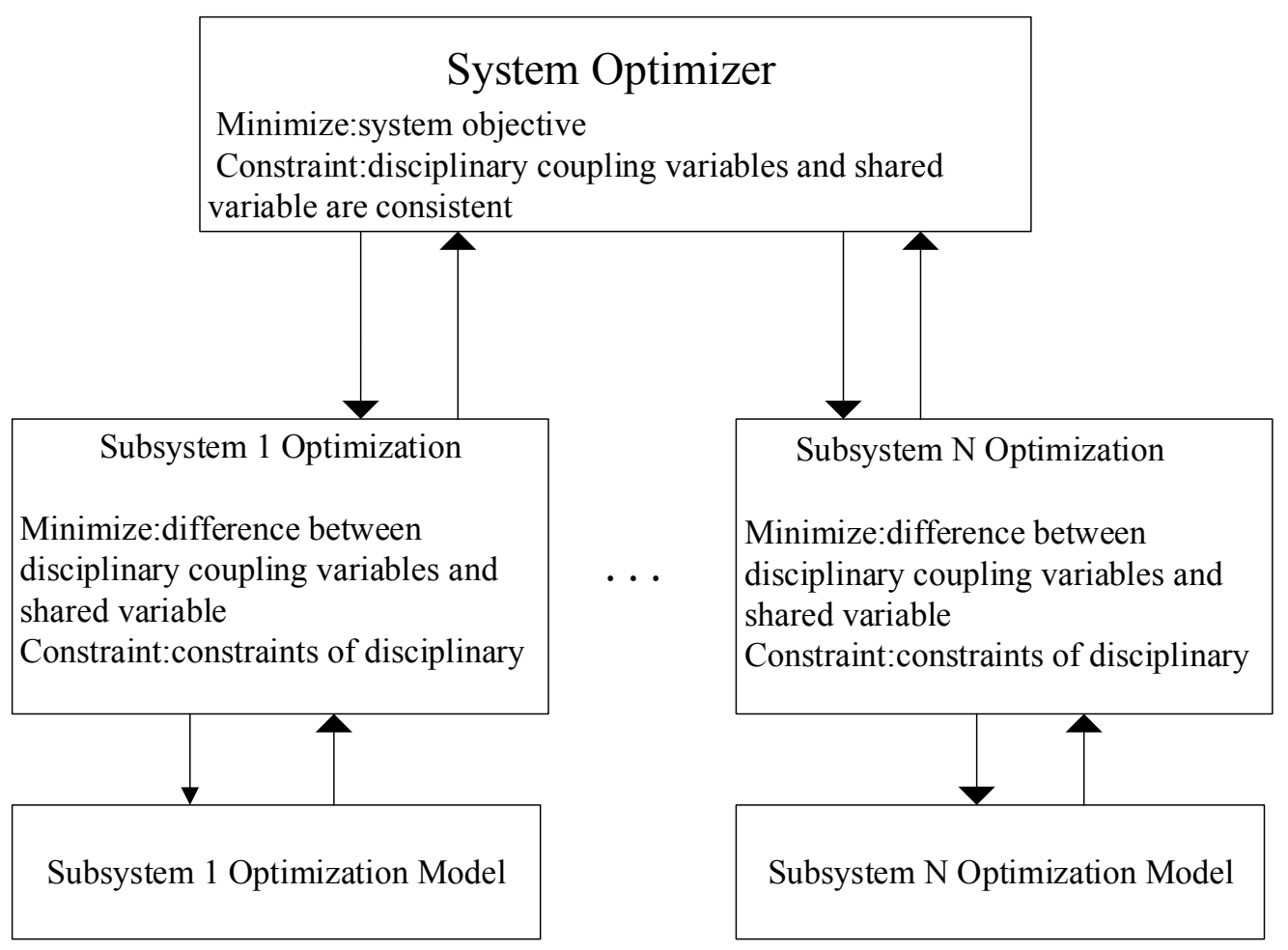

Fig. (5). Theory of collaborative optimization.

\section{$\bar{\sigma} \leq 276$}

Each plate thickness requires a certain range:

$10 \leq t_{i} \leq 30, i=1,2,4$

$20 \leq t_{i} \leq 55, i=3,5$

\subsubsection{Mathematical Model}

$$
\begin{cases}W(\vec{T}) & =\sum_{j=1}^{n} m_{j} \\ s . t \quad & \bar{\sigma} \leq 276 \\ & 10 \leq t_{i} \leq 30, i=1,2,4 \\ & 20 \leq t_{i} \leq 55, i=3,5\end{cases}
$$

\section{MULTI-DISCIPLINARY OPTIMIZATION (MDO)}

Here the author uses the Isight software on the work device multidisciplinary collaborative optimization. Isight is a powerful computer-aided optimization platform. Users can integrate and manage complex simulation process with Isight, utilizing a variety of optimization algorithms to automatically discover optimized solution [19].

\subsection{Procedure of Cooperated Optimization Algorithm}

Cooperated optimization algorithm (CO) is proposed as a multilevel optimization algorithm based on consistency constraint optimization algorithm by Kroo. CO is a two-stage hierarchical method as shown in Fig. (5). The top level is system optimizer which optimized multidisciplinary variable to meet compatibility of constraints between disciplines and minimized system objective. Every sub-system is optimized with the minimum mean square error method between subspace variable subset and subspace analysis of calculation results. System level design variables are solved with the subspace constraints. System level design variables are considered as a fixed value. The algorithm eliminates the complicated system analysis, every subsystem is parallel to the other.

Tasks are integrated by general component Simcode of Isight as shown in Fig. (6). The arrows in the figure show routes of optimization process and data transfer [20], wherein the calculator module is used to calculate parameters changing and tilt force multiplication coefficient in kinematics optimization process. Meanwhile, the simulation module is used to calculate the stress changing and quality changing in finite element analysis.

Since the entire optimization design needs to observe the acuity of all factors to the optimization goal, as well as considering complexity of the model and computational cost factors, this paper divided the entire Task components into two parts: DOE and Optimization.

\subsection{Multidisciplinary Optimization Mathematical Model}

In the light of mathematical model determined by kinematics and stiffness during single-disciplinary optimization, a multidisciplinary optimization model is constructed: 


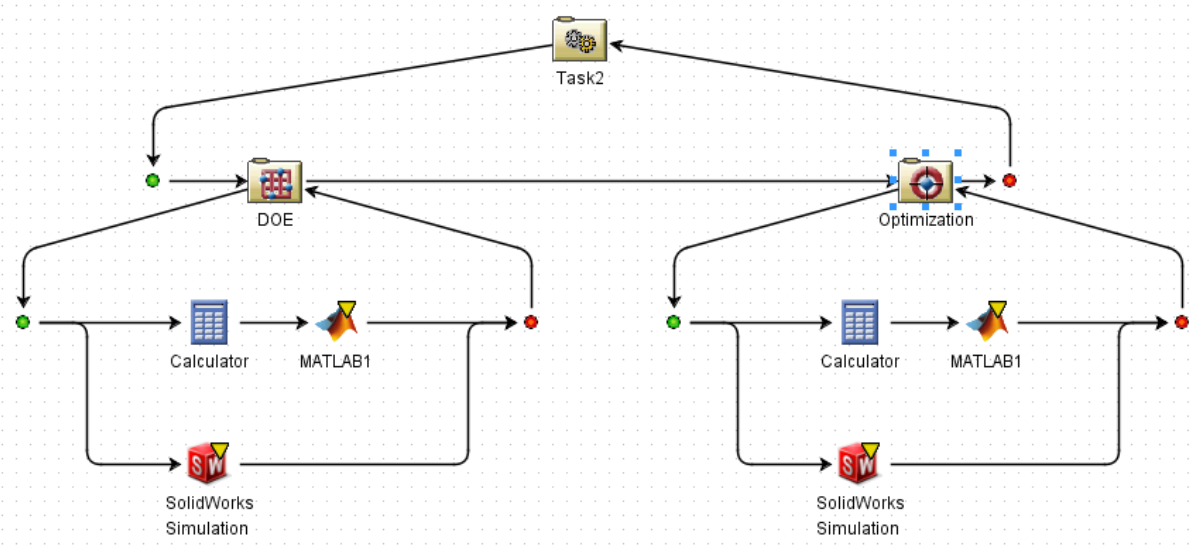

Fig. (6). Base procedure of multidisciplinary optimization based on Isight.

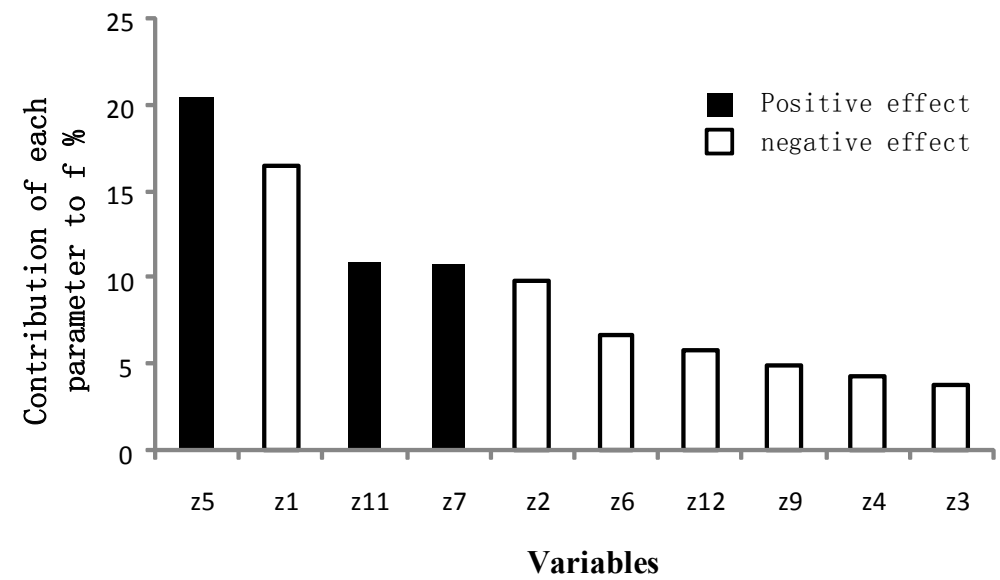

Fig. (7). Pareto of factors contribution to tilt force coefficient.

$$
\left\{\begin{array}{l}
\min F(\vec{X})=-K_{Z B} \\
W(\vec{T})=\sum_{j=1}^{n} m_{j} \\
\text { s.t } \quad g_{u}(\vec{X}) \leq 0 \quad(u=1,2,3, \ldots, 45) \\
\bar{\sigma} \leq 276 \\
10 \leq t_{i} \leq 30, i=1,2,4 \\
20 \leq t_{i} \leq 55, i=3,5
\end{array}\right.
$$

According to the requirement of optimization, $\mathrm{F}(\overrightarrow{\mathrm{X}})$ and $\mathrm{W}(\overrightarrow{\mathrm{T}})$ are chosen as the target parameters which respectively are tilt force multiplication coefficient and boomer mass. The optimization goal is to increase tilt force multiplication coefficient and reduce boomer mass as much as possible premising on the boundary condition requirement.

\subsection{Multidisciplinary Optimization Results and Analysis}

Integration platform is running. Before convergence it has went through several iterations.

Influence degree of each parameter for response is obtained from first conducted DOE experimental design, as shown from Fig. (7) to Fig. (8). In Fig. (7), it can be seen that $\mathrm{z}_{1}, \mathrm{z}_{2}, \mathrm{z}_{5}, \mathrm{z}_{7}, \mathrm{z}_{11}$ have greater contribution to tilt force multiplication coefficient. As can be seen from Fig. (8) that $t_{1}$ contributes more to boomer mass, while each hinge point coordinates contributes less, which is determined by form factors of the boom.

Feasibility of design point is determined from multiobjective optimization loop, obtaining collection of Pareto solutions and Pareto front graph, as shown in Fig. (9) and Fig. (10). Fig. (9) is historical chart of design feasibility in optimization process, where design feasibility value of each point provides a larger reference value for the outcome of the choice. It can be seen from the figure that in this article the optimization problem is mainly focused on the feasibility of the design point 7, and Pareto optimal solution software design point should be given in the selection.

Fig. (10) shows Pareto front graph formed from tilt force multiplication coefficient and mass of boomer. It is observed that tilt force multiplication coefficient and boomer mass are parameters directly proportional to each other, namely boomer mass increases with tilt force multiplication increases. Therefore, the best design point can be sought in area 


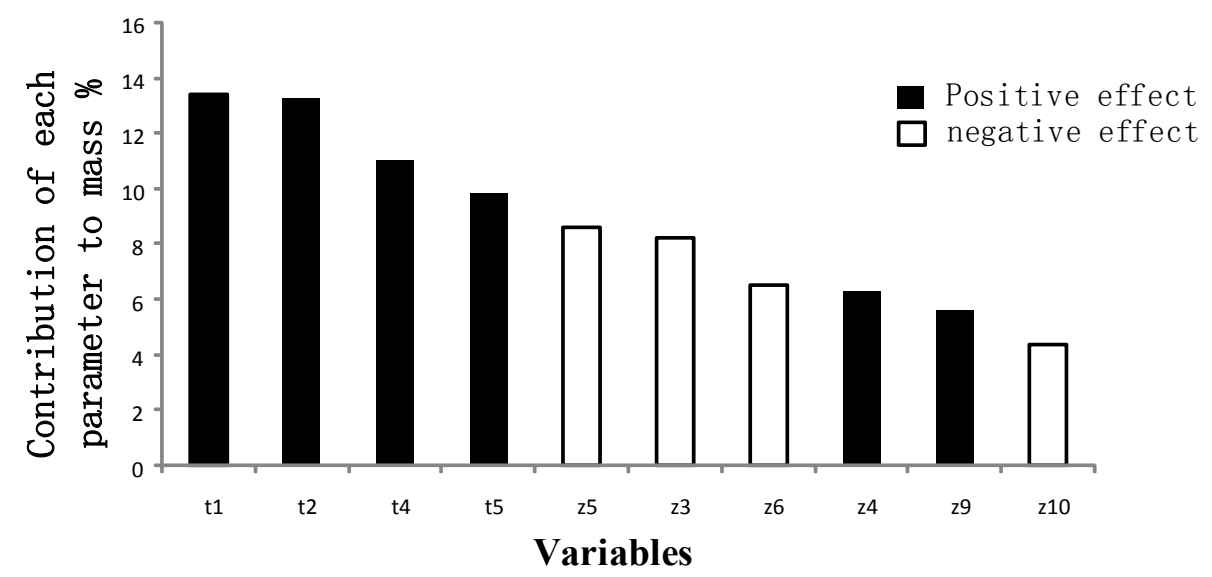

Fig. (8). Pareto of factors contribution to boomer mass.

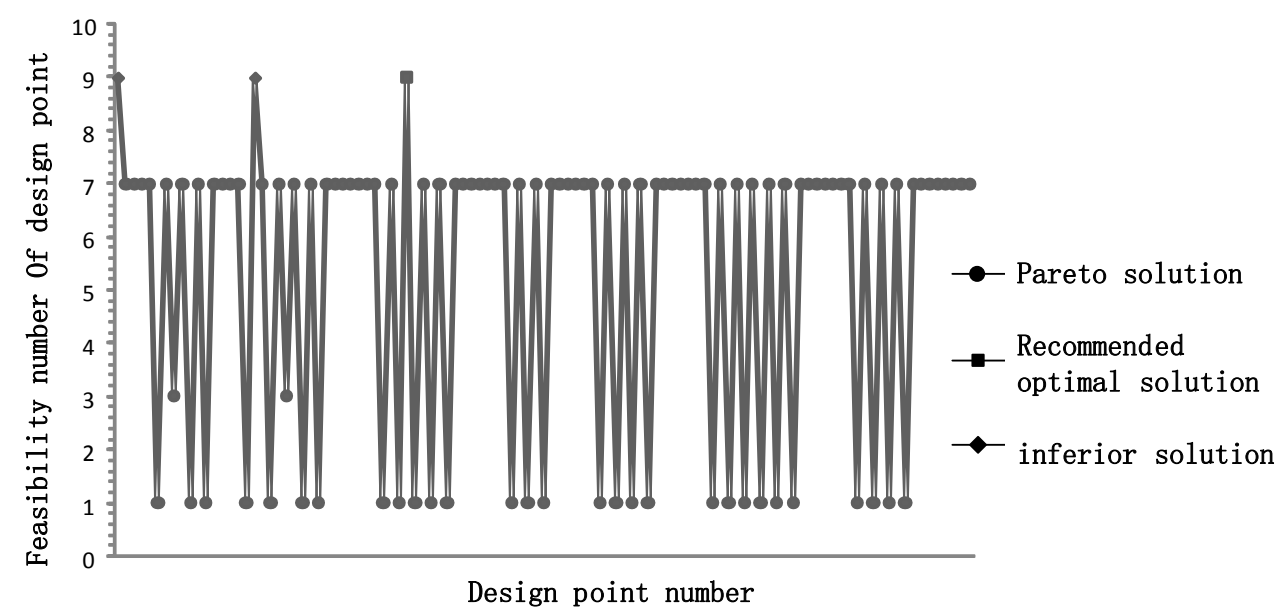

Fig. (9). Analysis chart of design feasibility.

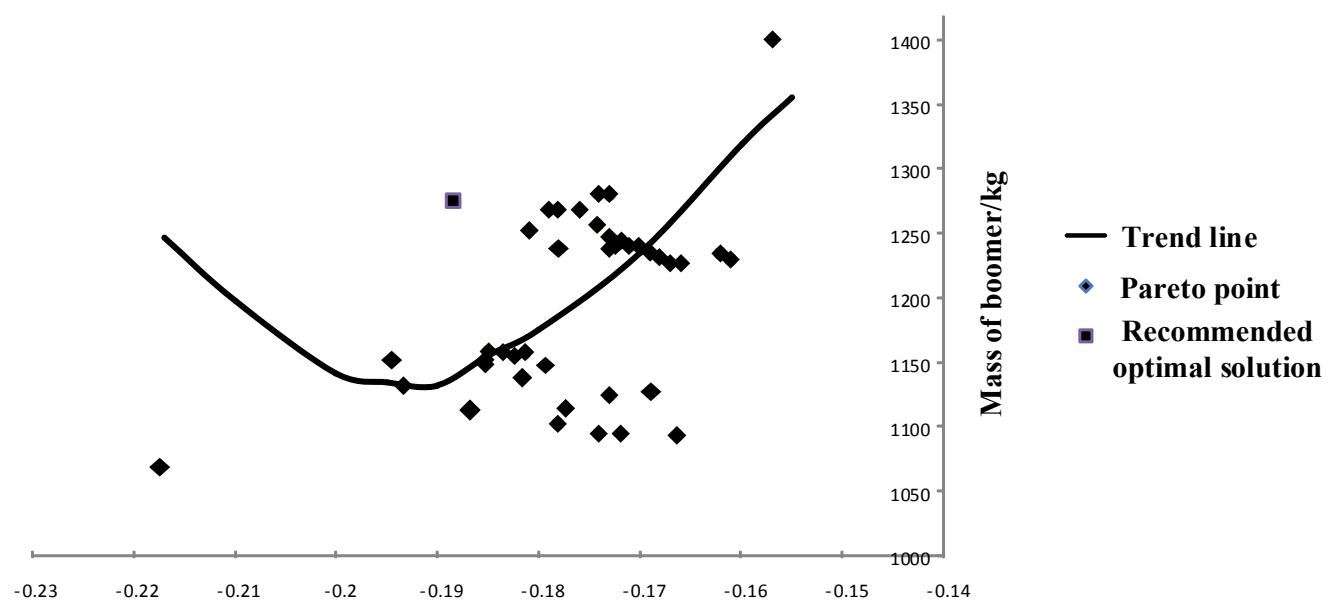

Tilt force multiplication coefficient

Fig. (10). Pareto front graph of force coefficient and boomer mass. 
Table 2. Results of multidisciplinary optimization.

\begin{tabular}{|c|c|c|c|c|}
\hline & Variables & Initial Value & Single-disciplinary & Multidisciplinary \\
\hline \multirow{6}{*}{ Kinematics } & $\mathrm{LGF} / \mathrm{mm}$ & 459.4 & 516.7 & 464.48 \\
\hline & $\mathrm{LEF} / \mathrm{mm}$ & 920 & 970.2 & 921.9 \\
\hline & $\mathrm{LGB} / \mathrm{mm}$ & 1304.5 & 945.7 & 1301.02 \\
\hline & $\mathrm{LBA} / \mathrm{mm}$ & 1904.2 & 2280.6 & 1922.03 \\
\hline & $\mathrm{LCB} / \mathrm{mm}$ & 767.9 & 707.8 & 777.1 \\
\hline & $\mathrm{LBE} / \mathrm{mm}$ & 807.3 & 1022.4 & 797.3 \\
\hline \multirow{5}{*}{ Stiffness } & $t_{1}$ & 25 & 18 & 15 \\
\hline & $\mathrm{t}_{2}$ & 25 & 20 & 15 \\
\hline & $t_{3}$ & 45 & 37 & 40 \\
\hline & $\mathrm{t}_{4}$ & 25 & 19 & 15 \\
\hline & $t_{5}$ & 41 & 44 & 21 \\
\hline \multicolumn{2}{|c|}{ Tilt Force $\mathrm{K}_{\mathrm{ZB}}$} & 0.153 & 0.172 & 0.187 \\
\hline \multicolumn{2}{|c|}{ Boomer Mass $/ \mathrm{kg}$} & 1997.5 & 1534.9 & 1257.8 \\
\hline \multicolumn{2}{|c|}{ Max Stress/MPa } & 204.23 & 229.91 & 263.96 \\
\hline
\end{tabular}

of $\mathrm{f}=19 \%$, where the best design points are also sought from this software.

The final optimization result is shown in Table 2. Compared to single-discipline optimization results, the bucket tilt force multiplication coefficient increased by $1.5 \%$ and boomer mass decreased by $18 \%$. Although the maximum equivalent stress increased a little, it is still in the safe zone. The optimization effect is obvious.

\section{CONCLUSION}

Based on the parametric model, kinematics and structural stiffness of single discipline analysis and optimization were made.

The optimization design platform of LHD is developed based on multidisciplinary and multi-objective soft Isight, considering requirements of kinematics discharge angle parallel transport and stiffness in the designing process of large underground LHD working device. The platform can consider coupling relationship among sub-disciplines, so that multidisciplinary collaborative optimization can be achieved.

By the multidisciplinary and multi-goals optimization platform based on Isight, parameter calculation, 3D modeling and finite element analysis in the loop from DOE to Optimization can be auto-executed, deducing duplication of work. At the same time, changing parameter of DOE and optimization can be monitored in real-time.

Contribution of parametric response can be obtained from Pareto figure of DOE which provides help to determine optimization parameter. Pareto front obtained from optimiza- tion can provide data support for choosing appropriate design point.

Single discipline optimization and multidisciplinary optimization were achieved separately. By comparing the optimization results, it can be seen that an optimized target compared to single discipline wasn't up to the optimal design but integrated optimization has a higher precision, and optimization effect is better due to coupling and conflict between sub-disciplines.

\section{CONFLICT OF INTEREST}

The authors confirm that this article content has no conflict of interest.

\section{ACKNOWLEDGEMENTS}

Fund program: National High Technology Research and Development Program of China (863 Program)(Grant No. 2011AA060403).

\section{REFERENCES}

[1] X. Wang, and D. Luo, "Linkage loader working device based on chaos genetic algorithm optimization design," Mining Machinery, vol. 9, no. 46, pp. 141-148, 2010.

[2] X. Bian, and F. Xiao, "Work device of the loader connecting rod type movable arm multi-objective optimization," Engineering Machinery, vol. 12, no. 13, pp. 76-86, 2002.

[3] X. Zhang, and X. Chen, "Three-dimensional modeling and finite element analysis of loader working device," Coal Mine Machinery, vol. 5, no. 105, pp. 51-59, 2014. 
[4] AIAA Multidisciplinary Design Optimization Technical Committee. Current State of the Art on Multidisciplinary Design Optimization(MDO), An AIAA White Paper 1911, vol. 3, no. 11, pp. 38-49, 2010.

[5] J. Sobieszczanski-Sobieski, "Optimization by decomposition: a step from hierarchic to non-hierarchic," NASA STI/Recon Technical Report, vol. 8, no. 1, pp. 51-78, 1988.

[6] J. Sobieszczanski-Sobieski, "The sensitivity of complex, internally coupled systems," AIAA Journal, vol. 28, no. 1, pp. 153-160, 1990

[7] K. Chiba, S. Obayashi, K. Nakahashi, and H. Morino, "Highfidelity multidisciplinary design optimization of wing shape for regional jet aircraft," In: Proceedings of the $23^{\text {rd }}$ AIAA Applied Aerodynamics Conference, vol. 5, no. 2, 2005, pp. 1012-1035.

[8] G. Cui, and H. Zhou, "Multi-objective optimization of 3-UPS-S parallel mechanism based on Isight," Transactions of the Chinese Society for Agricultural Machinery, vol. 44, no. 9, pp. 261-266, 2013.

[9] H. Yan, Simulation and Optimum of the Working Device of ZL80 Loader, University of Jilin, China, vol. 1, no. 1, pp. 1-89, 2006.

[10] W. J. Hu, and L. Chen, "Multidisciplinary design optimization for automobile disk brake based on Isight," Transactions of the Chinese Society for Agricultural Machinery, vol. 41, pp. 18-20, 2010.

[11] X. Li, and Y. Chen, "Wheel loader working device optimization design based on the sensitivity analysis," Advanced Materials Research, vol. 2, pp. 41-49, 2012.

[12] X. Zhang, X. Chen, and M. Yin, "Transmission box lightweight analysis based on ANSYS workbench," Mechanical Engineering \& Automation, vol. 5, pp. 57-59, 2010.
[13] X. K. Yin, Research on Kinematics and Dynamics about Reversesix-link-Mechanism of Working Device of Loader, University of Jilin, China, vol. 1, no. 1, pp. 1-78, 2011.

[14] Z. P. Zhao, Collaborative Optimization Research of the Wing Aerodynamic and Structural Analyses for an UAV Based on Isight, Nanjing University of Aeronautics and Astronautic, NanJing, vol. 1, no. 1, pp. 1-67, 2007.

[15] Q. S. Zhang, Ed., Research on Integration Technology and Application in the Davit Based on ISIGHT, South China University of Technology, ChangSha, vol. 1, no. 1, pp. 1-71, 2012.

[16] R. J. Balling, and J. Sobieszczanski-Sobieski, "An algorithm for solving the system-level problem in multilevel optimization," Structural and Multidisciplinary Optimization, vol. 9, pp. 168-177, 1995.

[17] J. P. Giesing, and M. Jean-Francois, A Summary of Industry MDO Applications and Needs, An AIAA White Paper, vol. 2, no. 1, pp. 128-137, 1998.

[18] Z. M. Yang, Z. M. Wang, and C. Q. Zhang, Wheel Loader, Chemical Industry Press, China, vol. 1, no. 1, pp. 63-64, 2006.

[19] J. Sobieszczanski-Sobieski, "The sensitivity of complex internally coupled systems," AIAA Journal, vol. 28, no. 1, pp. 153-160, 1990.

[20] M. Ebrahimi, M. R. Farmani, and J. Roshanian, "Multidisciplinary design of a small satellite launch vehicle using particle swarm optimization," Structural and Multidisciplinary Optimization, vol. 44, pp. 773-784, 2011

Received: September 16, 2014

Revised: December 23, 2014

Accepted: December 31, 2014

(C) Lei et al.; Licensee Bentham Open.

This is an open access article licensed under the terms of the Creative Commons Attribution Non-Commercial License (http://creativecommons.org/licenses/by$\mathrm{nc} / 4.0 /$ ) which permits unrestricted, non-commercial use, distribution and reproduction in any medium, provided the work is properly cited. 